\title{
New Phenotypic and Genotype SOD1 Mutation in Dominant Familial Motor Neuron Disease: A Case Report of a Family
}

\author{
Chandra $\mathrm{SR}^{1 *}$, Soumya $\mathrm{CV}^{2}$, Wahathule Rahul Raosaheb1, Malligurki Raghurama Rukmani ${ }^{3}$, Talakad N. Sathyaprabha ${ }^{3}$ and Anita $^{1}$
} Mahadevan $^{4}$

${ }^{1}$ Department of Neurology, National Institute of Mental Health and Neurosciences, Bengaluru ${ }^{2}$ Department of Neurology, AKG Hospital, Kannur, Kerala

${ }^{3}$ Department of Neuro-physiology, National Institute of Mental Health and Neurosciences, Bengaluru

${ }^{4}$ Department of Neuro-pathology, National Institute of Mental Health and Neurosciences, Bengaluru

\begin{abstract}
Anterior horn cell diseases are a group of pure motor disorders involving both upper and lower motor neurons. They are currently untreatable and carry variable course based on the phenotype. The nervous system is vulnerable for oxidative stress due to its high oxygen consumption, low antioxidants, poor regenerative capacity, and presence of metal ions. A 36-year-old female had exertion induced cramps of her right lower limb, coldness in the right lower limb, progressive weakness, wasting, and fasciculation; became bilateral within 4 years. She had cyanosis, hypothermia, and decreased sweating of right leg with psoriasis. Her nerve conduction and electromyography studies were suggestive of anterior horn cell disease, which was supported by histopathology. She had severe reduction in total volume of sweat produced and prolonged sweat latency on the right-sided limbs as assessed by Quantitative sudomotor axon reflex test. DNA testing showed SOD1 cytogenetic band exon 4 of the SOD1 gene. chr. 21:33039650c $>C / T$; c. 319c>C/T. We report a new phenotype in dominantly inherited amyotrophic lateral sclerosis, with asymmetrical vasomotor and sudomotor changes and psoriasis.
\end{abstract}

Keywords: Amyotrophic lateral sclerosis; New SOD1 mutation; Psoriasis; Sweating; Thermoregulation

\section{Introduction}

Charles Bell in 1824 was the first to describe amyotrophic lateral sclerosis (ALS). Later in 1860, Jean-Martin Charcot and Marie described amyotrophy with spasticity, fibrillation, dropped heads, and childish behavior [1]. Motor neuron disorders are neuronopathies, which are slowly progressive pure motor disorders; which can be symmetrical, asymmetrical, proximal, distal, with or without the involvement of cranial neurons. Based on the heritability, it is classified as familial ALS or sporadic ALS. Based on the regions affected, it is classified as lower motor neuron, upper motor neuron, or combined. There is a lack of uniformity in the phenotype. However, it has key features which suggest the common diagnosis. The first categorization of these common features was done by Dr. Edward Lambert in 1957. This was revised by El-Escoril and Awaji [2,3]. Later, all the available criteria were clubbed to form the revised El-Escoril criteria by Brooks et al. in the year 2000 .

\section{Case Presentation}

\section{Different patterns and associations of anterior horn cell dis-} ease

The patterns are categorized into asymmetrical distal weakness without a sensory loss (NP5). This includes progressive muscular atrophy (PMA), primary lateral sclerosis, ALS. Next pattern is a symmetric weakness without a sensory loss (NP7), which includes spinal muscular atrophy (SMA), PMA; and differential diagnosis is Charcot Marie Tooth which is NP2 and hereditary motor neuropathy. The next pattern is focal midline proximal symmetric (NP8) under this subtype patterns MP6 if neck and trunk are affected, MP7 if bulbar. They can present as pure lower motor, mixed upper and lower motor, pure upper motor. The well-known associations are cognitive deficits, behavioral symptoms, psychiatric symptoms, and Parkinsonian features.

\section{Familial amyotrophic lateral sclerosis}

ALS is found to be familial in about $10 \%$ of patients [4]. Familial aggregation is seen, mostly indicating dominant inheritance. SODI gene, ALS1, HNRNPA1, ALS20, MATR3, ALS21, OPTN, ALS12, PFN1, ALS18, SETX, ALS4, SIGMAR!, ALS!^, SPG11, SQSTM1, TARDBP, ALS10, TBK1, TUBA4A, ALS22<UBQLN2, ALS22, UBQLN2, ALS15, $V A P B, A L S 8, V C P, A L S 14$ and several other genes are identified [5]. SOD1 antisense oligonucleotide therapy, small molecule gene therapy targeting expression of C90RF72 repeat expansion is being tried based on the genetic information obtained [6]. The familial patients were clinically identified based on the positive family history of similar illness or dementia [7].

\section{Clinical features}

Our index patient is a 36-year-old female, who had presented with exertion induced cramps in the whole of right lower limb and weakness of 4 years duration in the form of difficulty in holding chappals in right lower limb, high-stepping, falling with an injury to toes. These symptoms were slowly progressive. She developed twitching of muscles in both thighs, buckling and difficulty in climbing over the next 2 years and became wheelchair bound at the time of admission to our institution. Examination showed a well-nourished patient with the normal HMSE score. Her cranial nerves and upper limbs were

*Corresponding author: Chandra SR, Department of Neurology, National Institute of Mental Health and Neurosciences, Bangalore, India, Tel: 9449106799 E-mail: drchandrasasi@yahoo.com

Received May 16, 2018; Accepted June 25, 2018; Published June 28, 2018

Citation: Chandra SR, Soumya CV, Raosaheb WR, Rukmani MR, Sathyaprabha TN, et al. (2018) New Phenotypic and Genotype SOD1 Mutation in Dominant Familial Motor Neuron Disease: A Case Report of a Family. J Neurol Disord 6: 383. doi:10.4172/2329-6895.1000383

Copyright: (c) 2018 Chandra SR, et al. This is an open-access article distributed under the terms of the Creative Commons Attribution License, which permits unrestricted use, distribution, and reproduction in any medium, provided the original author and source are credited. 
normal. On the right side, both dorsal and plantar flexors and small muscles were grade 0 . Knee flexors were grade 3 and extensors were grade 4. Muscles at the hip were grade 4 in all groups and grade 3 in extensors. There was wasting of glutei, quadriceps, calf muscles, and small muscles on the right side. Wasting was also noticed in the left thigh. Fasciculation's were seen in quadriceps on both sides, moreover right, and occasionally over the calf. Right ankle jerk was absent, and other reflexes in the right lower limb were elicit able. On the left side, it was normal. Plantar response was absent. In addition, the patient had cyanosis in the right toes, loss of hair, dry skin, and exfoliation of skin in fingers, which was diagnosed as psoriasis and was being treated. The temperature was measured using the ordinary thermometer and found to be as follows: left axilla - 98.6; Right axilla - $98.4^{\circ} \mathrm{F}$; left leg $-93.2^{\circ} \mathrm{F}$ and right leg $-91.1^{\circ} \mathrm{F}$.

A five-generation family tree was drawn (Figure 1). Details about the first generation were not available. Second generation had 3 females. All of them had a similar illness and died before 45 years, and cause of death was respiratory distress. In the 3 rd generation, 3 females and 1 male were affected, and all of them died very young. Our index case belongs to 4 th generation. Her younger brother, aged 32 years, had a very rapidly progressive course but with similar phenotype involving right lower limb, cyanosis, trophic changes, and psoriasis followed by respiratory distress without significant generalization. He was treated with immunomodulators with the diagnosis of rapidly progressive anterior horn cell disease. The patient was the youngest to die in the group with wasting of muscles (Figure $2 a$ ) and psoriasis. Another younger brother of our index case is wheelchair bound. She has two daughters aged 16 years; 5 years and one son aged 13 years. Interestingly, most of the family members, who did not have neurological manifestations, also had psoriasis (Figure $2 \mathrm{~b}$ ).

\section{Investigations}

Complete hemogram, liver function test, renal function test, thyroid function test, serum electrolytes, mitochondrial function, serum copper, serum ceruloplasmin, Bence Jones protein in the urine, serum Vitamin B12, Rheumatoid arthritis factor and antineuronal antibodies were all normal in the indexed patient. She was negative for HIV. Biopsy from left quadriceps revealed preserved fascicular architecture. There was variation in fiber size, rounding, groups of angulated fibers with clumped nuclei, and no inflammation. Modified Gomori Trichrome staining of the muscle showed greenish condensed staining material in hypertrophied fibers suggestive of target fibers. There were no ragged red fibers. There was grouping of Type 1 and Type 2 fibers. Type 1 fibers were atrophied. There were No Cytochrome C Oxidase-deficient fibers. Neurogenic atrophy with denervation and reinnervation suggestive of anterior horn cell disease was observed (Figures $2 c$ and $2 d$ ). Resting heart rate variability and conventional cardiac autonomic function testing (Ewing's protocol), which measures heart rate and blood pressure changes to certain physiological maneuvers such as deep breathing, valsalva maneuver, isometric handgrip, and orthostasis, also showed normal cardiac autonomic function in her. Quantitative sudomotor axon reflex test (QSART) test was carried out in the index case, two of her symptomatic cousins and her two asymptomatic children. It was normal in her daughter whose genetic testing was negative for the SOD1 mutation (Figure 3a). There was a similarity and absent sweat response in the right forearm and right foot in the test results of QSART of the index case, symptomatic cousins and her asymptomatic son, whose genetic testing showed SOD1 mutation. They showed a reduction in the total volume of sweat produced in the right proximal leg and right distal leg; prolonged sweat response latency in right distal leg. and absent sweat response in the right forearm and right foot (Figure $3 b$ ).

Nerve conduction study showed normal latency and normal conduction velocity, in both motor and sensory nerves of bilateral lower limbs and upper limbs. "F" latency was also normal. However, there was a reduction in the amplitude of motor nerves of the lower limbs (Table 1).
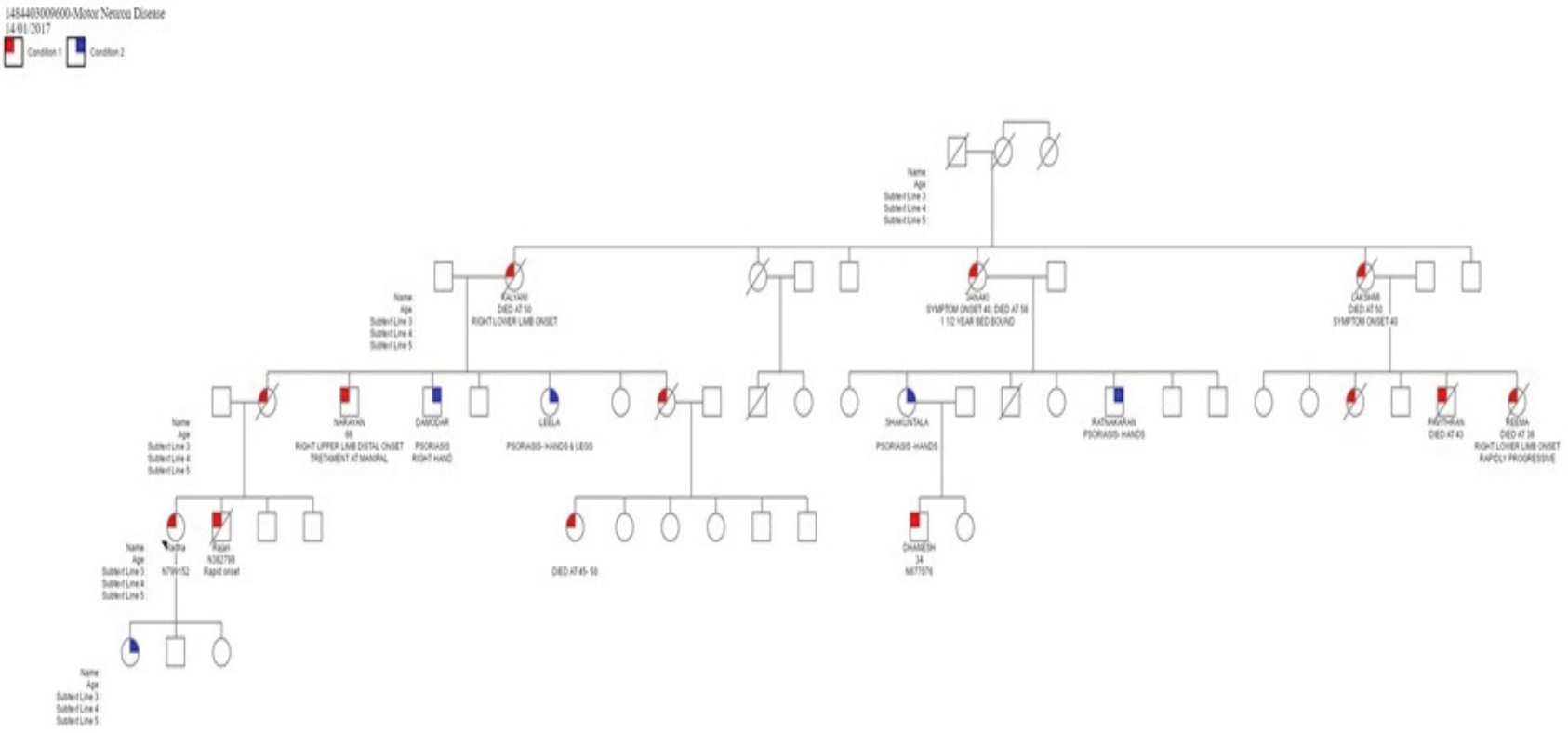

\section{Condition 1-MND like features; Condition 2- Psoriasis}

Figure 1: Pedigree chart showing the distribution of disease in the family. 
Citation: Chandra SR, Soumya CV, Raosaheb WR, Rukmani MR, Sathyaprabha TN, et al. (2018) New Phenotypic and Genotype SOD1 Mutation in Dominant Familial Motor Neuron Disease: A Case Report of a Family. J Neurol Disord 6: 383. doi:10.4172/2329-6895.1000383
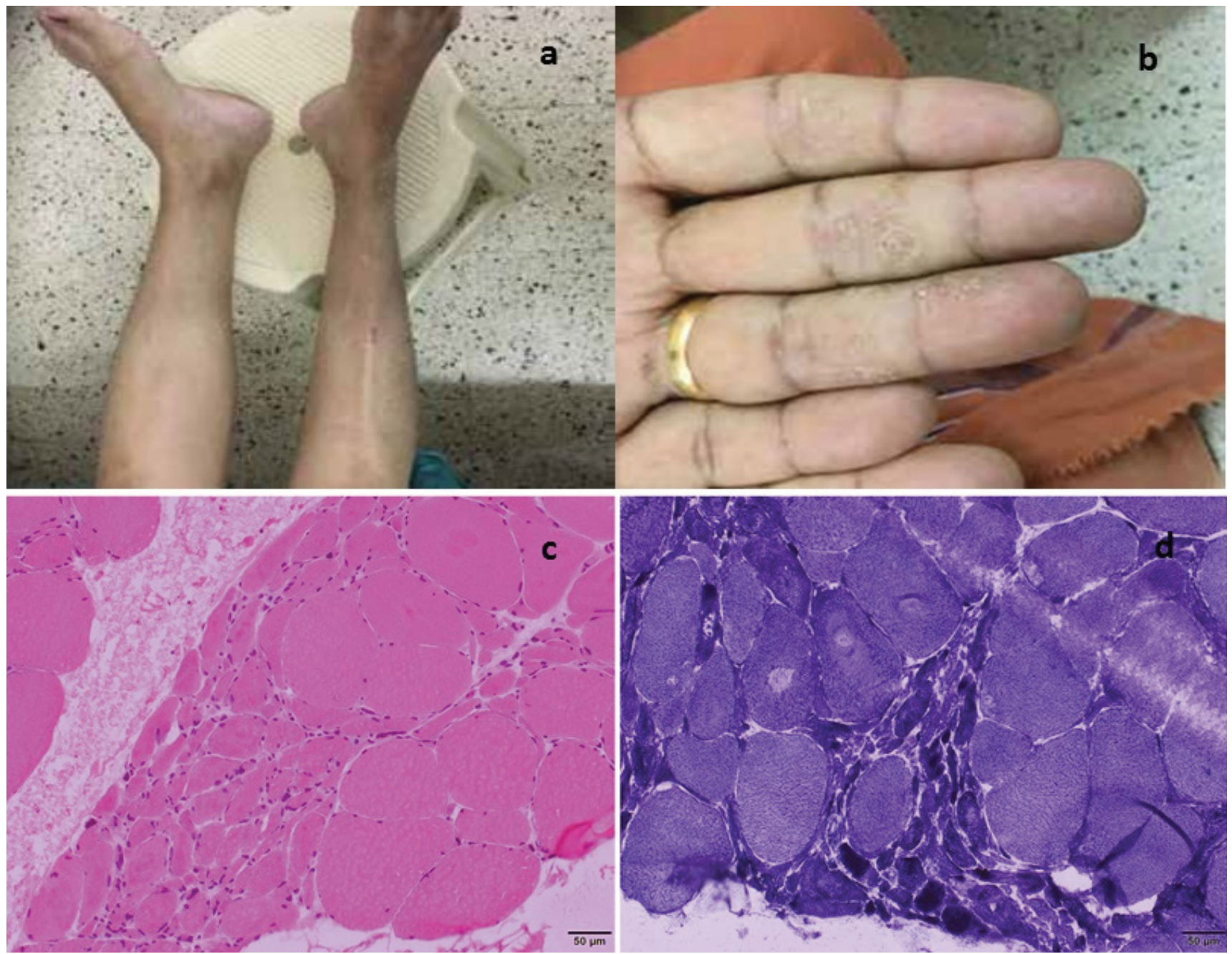

Figure 2 (a) Muscle wasting in the leg.; (b) Psoriasis; (c) Microphotograph of muscle showing collapsed fascicles with atrophic fibers and groups of large hypertrophic fibers; (d) Group atrophy.

\section{DNA test}

The variant analysis was performed by sanger sequencing based on SOD 1 gene with reference sequence nomenclature NG_008689.1. Region 5001.14310 in the NCBI gene bank database and the cDNAbased allele (c) for the variations and the corresponding amino acids change based on SOD1 RefSeq sequence ENST00000270142. Exon 4 of SOD1 gene was PCR amplified, and the product was sequenced. It was aligned to available reference sequence mentioned above (NG_008689.1. Region 5001.14310) to detect variations using variant analysis software program. A heterozygous missense variation in exon 4 of the SOD1 gene, chr. 21:33039650c $>C / T ; c .319 c>C / T$ that results in the amino acid substitution of phenylalanine for leucine at codon 107 (p. Leu107Phe) was detected in our index case. This was further validated by Sanger sequencing (Figure 4). This SOD1 mutation was detected heterozygous in the affected cousins of the patient and unaffected son. However, QSART test was abnormal in the son who was otherwise clinically normal bur carrying the heterozygous mutation.

\section{Discussion}

Superoxides such as reactive oxygen species, superoxide radicals, hydrogen peroxide, hydroxyl radicles, and single oxygen have a very short half-life as they are extremely active and postulated to be involved in several neurodegenerative diseases. There are naturally occurring scavengers which when imbalanced results in tissue damage. Superoxide dismutase catalyzes the conversion of reactive oxygen into hydrogen peroxide [8]. There are three types of dismutases: superoxide dismutase SOD1 otherwise called $\mathrm{Cu}-\mathrm{Zn}$ Sod present in the cytosol, nuclei and mitochondrial matrix; $S O D 2$ or manganese superoxide dismutase found in the mitochondrial matrix, and SOD 3 or extracellular-superoxide dismutase, constituted by a complex of $\mathrm{Cu}$ and $\mathrm{ZN}$ and is seen in extracellular space [9]. Oxidative stress occurs due to an imbalance in oxidant and antioxidant homeostasis. Suboptimal levels of reactive oxygen species serve (ROS) as a cell signaler, but unregulated ROS causes toxicity $[10,11]$. The nervous system is vulnerable because of high oxygen consumption, low antioxidant levels, low regenerative capacity, and high levels of metal ions to maintain function; which will cause ROS generation. These act on unsaturated lipids, proteins and DNA. Lipid peroxidation causes breakdown products such as 4 (oh) 2.3-nonenal, acrolein, Malondialdehyde, and F2-isoprostanes which inhibit Glucose transporter type 3, GLT1, Na+ K+ Atpase and activate apoptotic protein kinases. Interaction with DNA causes mutations 
Citation: Chandra SR, Soumya CV, Raosaheb WR, Rukmani MR, Sathyaprabha TN, et al. (2018) New Phenotypic and Genotype SOD1 Mutation in Dominant Familial Motor Neuron Disease: A Case Report of a Family. J Neurol Disord 6: 383. doi:10.4172/2329-6895.1000383

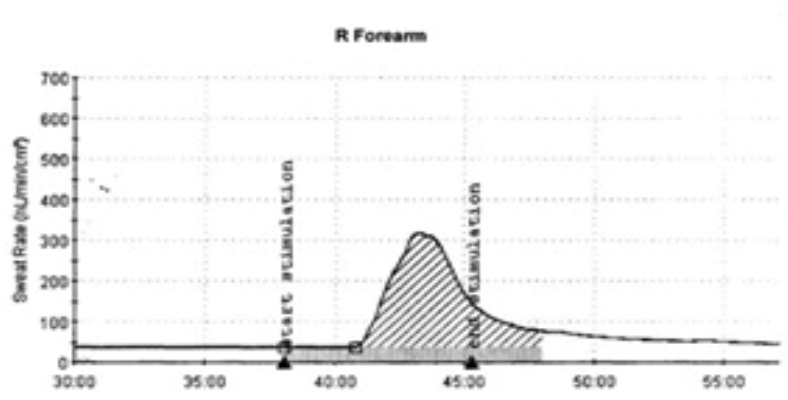

a
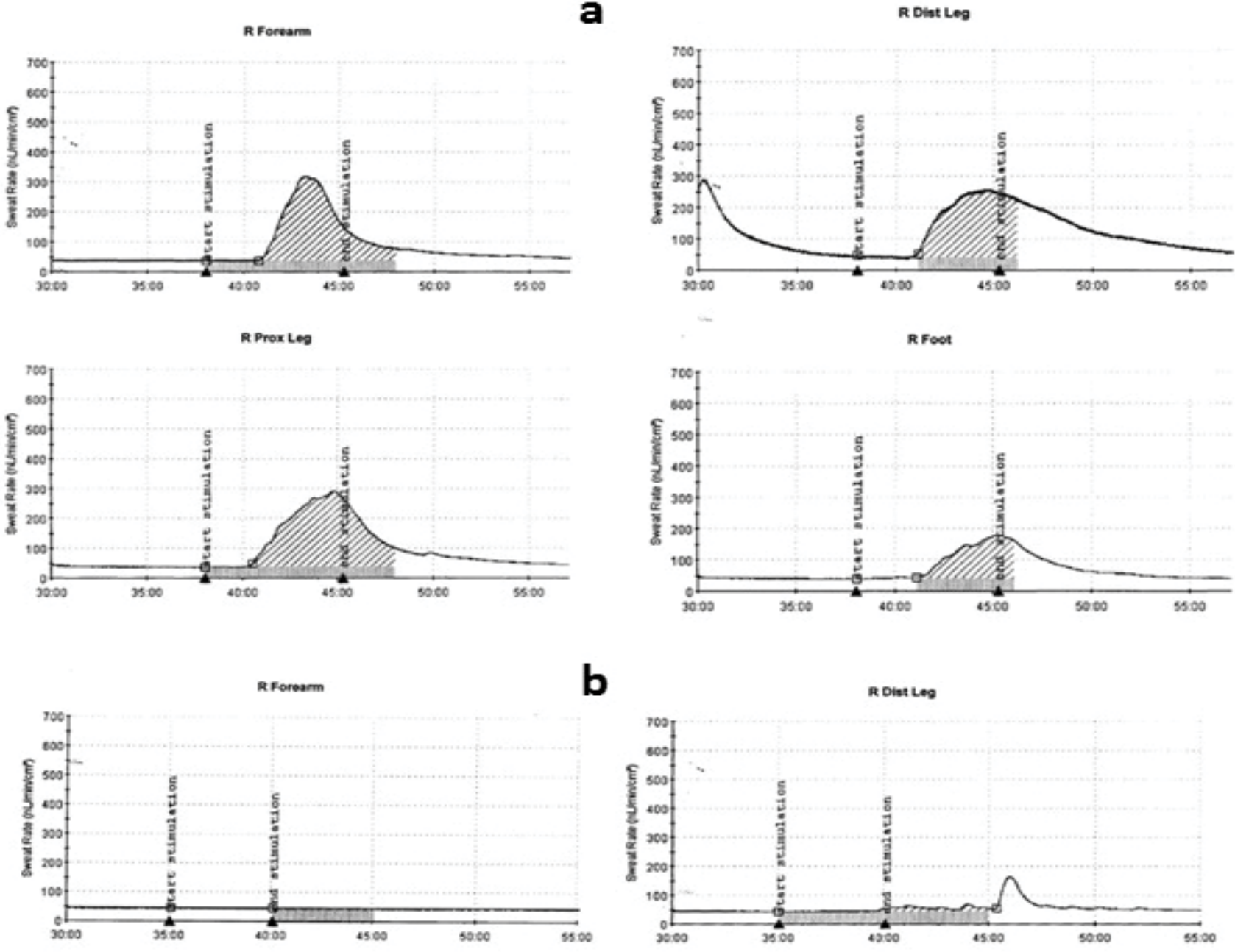

b
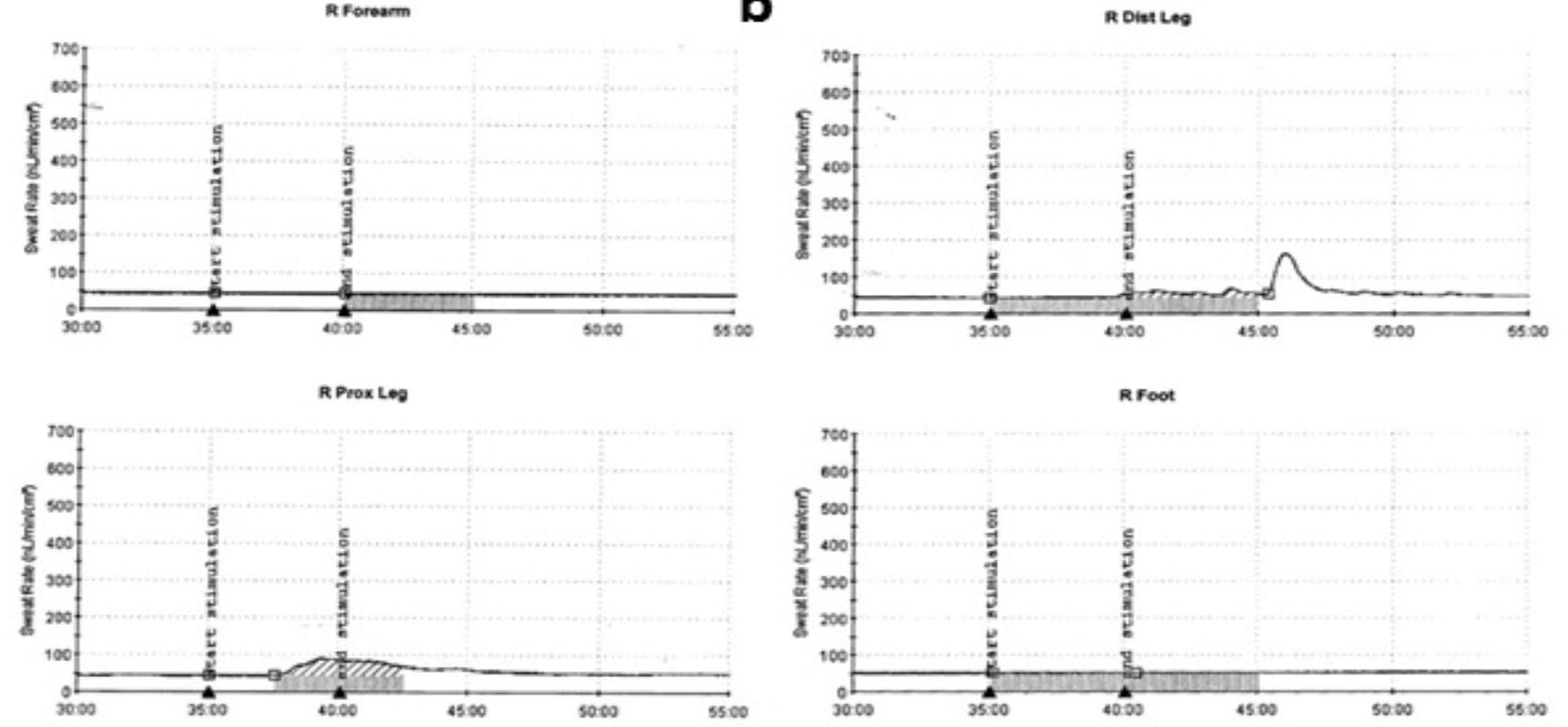

Figure 3: (a) Normal QSART in unaffected daughter; (b) Abnormal QSART in the asymptomatic son with SOD1 mutation.

\begin{tabular}{|c|c|c|c|c|c|}
\hline \multicolumn{6}{|c|}{ Motor Nerve Studies } \\
\hline Nerve & Site & Lat1 (ms) & Dur (ms) & $\operatorname{Amp}(\mathrm{mV})$ & $\operatorname{NCV}(\mathrm{m} / \mathrm{s})$ \\
\hline \multirow{4}{*}{ Median } & Rt. Wrist & 3.02 & 11.51 & 12.4 & \multirow{2}{*}{55.56} \\
\hline & Rt. Elbow & 6.98 & 9.69 & 11.1 & \\
\hline & Lt. Wrist & 2.81 & 11.35 & 11.4 & \multirow{2}{*}{58.67} \\
\hline & Lt. Elbow & 6.56 & 11.35 & 11.1 & \\
\hline \multirow{4}{*}{ Ulnar } & Rt. Wrist & 2.40 & 12.19 & 1.08 & \multirow{2}{*}{65.93} \\
\hline & Rt. Elbow & 6.04 & 11.88 & 9.3 & \\
\hline & Lt. Wrist & 2.40 & 11.77 & 10.5 & \multirow{2}{*}{67.80} \\
\hline & Lt. Elbow & 5.94 & 11.98 & 9.8 & \\
\hline
\end{tabular}




\begin{tabular}{|c|c|c|c|c|c|}
\hline \multirow{4}{*}{ Peroneal } & Rt. Ankle & 4.79 & 8.54 & $2.0^{*}$ & \multirow{2}{*}{47.95} \\
\hline & Rt. Knee & 11.88 & 9.06 & $1.2^{*}$ & \\
\hline & Lt. Ankle & 4.17 & 9.17 & 5.1 & \multirow{2}{*}{54.40} \\
\hline & Lt. Knee & 10.42 & 9.9 & 3.5 & \\
\hline \multirow{4}{*}{ Tibial } & Rt. Ankle & 5.21 & 10.10 & $0.9^{*}$ & \multirow{2}{*}{60.26} \\
\hline & Rt. Knee & 11.35 & 12.60 & $0.6^{*}$ & \\
\hline & Lt. Ankle & 2.92 & 11.35 & 8.1 & \multirow{2}{*}{49.33} \\
\hline & Lt. Knee & 10.42 & 12.40 & 6.6 & \\
\hline \multicolumn{6}{|c|}{ Sensory Nerve Studies } \\
\hline Nerve & Site & Lat1 (ms) & Dur (ms) & $\operatorname{Amp}(\mu \mathrm{V})$ & $\mathrm{NCV}(\mathrm{m} / \mathrm{s})$ \\
\hline \multirow{2}{*}{ Median } & Rt. Wrist & 2.75 & 1.21 & 35.3 & 54.55 \\
\hline & Lt. Wrist & 2.67 & 1.21 & 42.0 & 56.18 \\
\hline \multirow{2}{*}{ Ulnar } & Rt. Wrist & 2.25 & 1.25 & 32.1 & 57.78 \\
\hline & Lt. Wrist & 2.33 & 1.13 & 37.5 & 55.79 \\
\hline \multirow{2}{*}{ Sural } & Rt. Mid-Calf & 2.75 & 2.13 & 23.9 & 50.91 \\
\hline & Lt. Mid-Calf & 2.71 & 2.13 & 21.6 & 51.66 \\
\hline
\end{tabular}

Table 1: Nerve conduction study of the index case.

a

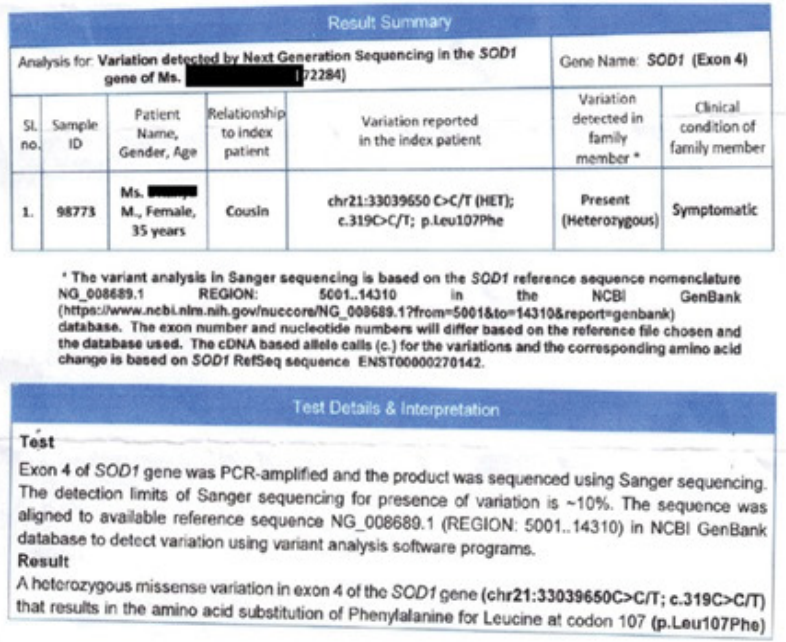

b

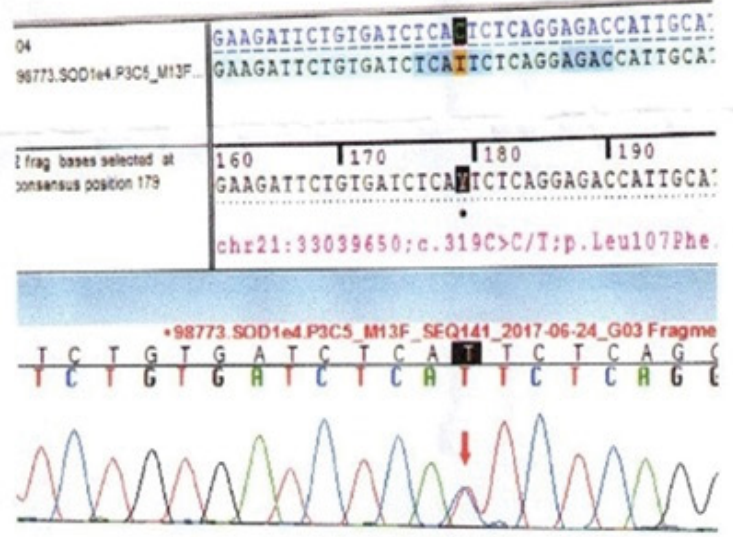

Figure 4: (a) DNA Test result; (b) Gel picture.

[12]. ALS results from gain of function due to aggregation of misfolded form of SOD and its conversion to a pro-oxidant protein [13].

\section{Psoriasis and neurological diseases}

Apart from the direct complications of psoriasis induced skeletal changes, an association with Parkinson's disease is reported based on a population-based study which was not supported by other studies and no other association is reported to our knowledge [14].

\section{Unique features of this amyotrophic lateral sclerosis family}

Right lower limb was the first affected part in all the all affected members of this family. Vasomotor and sudomotor changes were also seen in the right lower limb. They had associated psoriasis. Death was due to respiratory distress and occurred early. Even the clinically asymptomatic son of the patient showed abnormality in QSART test involving his right-sided limbs. It is possible that the right side being dominant and involved in more work than the left it became vulnerable. Any other unique nature of the anterior horn cells and intermediolateral grey needs to be understood.

\section{Conclusion}

We report an index case and her family with a unique phenotype of ALS, for which the authors could not find any earlier reference in the literature. Vasomotor and sudomotor changes on the right-sided limbs were observed in both symptomatic and asymptomatic family members with the heterozygous gene mutation. They also had psoriasis and early death. The current SOD1 heterozygous mutation seen in all affected persons also is not reported to the best of our knowledge. The QSART abnormalities seen in the child with no clinical symptoms indicate this could be an early biomarker for persons with this mutation. This unique combination needs further study to explore new treatment options other than antioxidants, antiexcitotoxins and caloric restriction currently practiced, if its pathomechanisms are identified.

\section{Limitations}

We did not do the SOD1 mutation analysis in family members who had psoriasis but no evidence of MND. We could not exactly establish the pathogenesis of this phenotype with the available tools. 
Citation: Chandra SR, Soumya CV, Raosaheb WR, Rukmani MR, Sathyaprabha TN, et al. (2018) New Phenotypic and Genotype SOD1 Mutation in Dominant Familial Motor Neuron Disease: A Case Report of a Family. J Neurol Disord 6: 383. doi:10.4172/2329-6895.1000383

\section{Declaration of Patient Consent}

The authors certify that they have obtained all appropriate patient consent forms. In the form the patient(s) has/have given his/her/their consent for his/her/their images and other clinical information to be reported in the journal. The patients understand that their names and initials will not be published, and due efforts will be made to conceal their identity, but anonymity cannot be guaranteed.

\section{References}

1. Goetz CG (2000) Amyotrophic lateral sclerosis: Early contributions of JeanMartin Charcot. Muscle Nerve 23: 336-343.

2. Brooks BR (1994) El Escorial world federation of neurology criteria for the diagnosis of amyotrophic lateral sclerosis. Subcommittee on motor neuron diseases/Amyotrophic lateral sclerosis of the world federation of neurology research group on neuromuscular diseases and the El Escorial "Clinical limits of amyotrophic lateral sclerosis" workshop contributors. J Neurol 124: Suppl 96-107.

3. Brooks BR, Miller RG, Swash M, Munsat TL (2000) World Federation of Neurology Research Group on Motor Neuron Diseases. El Escorial revisited: Revised criteria for the diagnosis of amyotrophic lateral sclerosis. Amyotroph Lateral Scler Other Motor Neuron Disord 1: 293-299.

4. Plato CC, Reed DM, Elizan TS, Kurland LT (1967) Amyotrophic lateral sclerosis-Parkinsonism-dementia complex of Guam. IV. Familial and genetic investigations. Am J Hum Genet 19: 617-632.
5. Byrne S, Bede P, Elamin M, Kenna K, Lynch C, et al. (2011) Proposed criteria for familial amyotrophic lateral sclerosis. Amyotroph Lateral Scler 12: 157-159.

6. Miller TM, Pestronk A, David W, Rothstein J, Simpson E, et al. (2013) An antisense oligonucleotide against SOD1 delivered intrathecally for patients with SOD1 familial amyotrophic lateral sclerosis: A phase 1, randomised, first-inman study. Lancet Neurol 12: 435-442.

7. Hardiman O, Van den Berg LH, Kiernan MC (2011) Clinical diagnosis and management of amyotrophic lateral sclerosis. Nat Rev Neurol 7: 639-649.

8. McCord JM, Fridovich I (1969) Superoxide dismutase. An enzymic function for erythrocuprein (hemocuprein). J Biol Chem 244: 6049-6055.

9. Okado-Matsumoto A, Fridovich I (2001) Subcellular distribution of superoxide dismutases (SOD) in rat liver: $\mathrm{Cu}, \mathrm{Zn}-\mathrm{SOD}$ in mitochondria. J Biol Chem 276 38388-38393.

10. Neill S, Desikan R, Hancock J (2002) Hydrogen peroxide signalling. Curr Opin Plant Biol 5: 388-395.

11. Halliwell B, Gutteridge JM (1985) Free Radicals in Biology and Medicine. Free Radic Biol Med 1: 331-332.

12. McCord JM (1994) Mutant mice, $\mathrm{Cu}, \mathrm{Zn}$ superoxide dismutase, and motor neuron degeneration. Science 266: 1586-1587.

13. Barnham KJ, Masters CL, Bush Al (2004) Neurodegenerative diseases and oxidative stress. Nat Rev Drug Discov 3: 205-214.

14. Sheu JJ, Wang KH, Lin HC, Huang CC (2013) Psoriasis is associated with an increased risk of Parkinsonism: A population based 5-year follow-up study. $J$ Am Acad Dermatol 68: 992-999. 